\title{
Destination-Based HoL Blocking Elimination
}

\author{
T. Nachiondo, J. Flich, and J. Duato \\ Dept. of Computer Engineering \\ Universidad Politécnica de Valencia \\ Camino de Vera, 14, 46071-Valencia, Spain \\ \{tnachion,jflich,jduato\}@ disca.upv.es
}

\begin{abstract}
In future interconnection networks, congestion management is likely to become a critical issue owing to increasing power consumption and cost concerns. As congested packets introduce head-of-line ( $\mathrm{HoL})$ blocking to the rest of packets, congestion spreads quickly. The best-known solution to HoL blocking, Virtual Output Queues (VOQs), is not scalable at all or too costly when implemented in large networks. In previous works, we proposed an efficient and costeffective solution, referred to as Destination-Based Buffer Management (DBBM). DBBM groups destinations into different sets, and packets addressed to destinations in the same set are mapped to the same queue. DBBM eliminates most of the HoL blocking (among packets addressed to different sets). It achieves very good results in terms of scalability, throughput, and robustness. However, depending on the distribution of packet destinations, it may introduce an uncertain degree of unfairness among packets mapped on the same queue.

In order to overcome this problem we propose the Dynamic DBBM mechanism (DDBBM). DDBBM dynamically eliminates completely the HoL blocking. Performance results show that DDBBM keeps (and in some cases improves) the good results achieved by DBBM in terms of throughput and scalability. Moreover, DDBBM solves the unfairness introduced by DBBM. As an example of applicability, in this paper we show that DDBBM can be applied to InfiniBand with no hardware modification.
\end{abstract}

\section{Introduction}

Many compute-intensive applications (nuclear weapon simulations, protein folding, global climate modeling, etc.) require continued research and technology development to deliver computers with steadily increasing computing power. The required levels of computing power can only be achieved with massively parallel computers. Examples of these systems are the Earth Simulator [9] and the Blue-
Gene/L [5]. In these systems, interconnection networks (ICTNs) with low communication latencies and high bandwidth are becoming a key component.

Also, internet portal servers and data-center servers are emerging environments where high performance ICTNs (InfiniBand [13], Myrinet [6] ...) are being preferred to build cluster-based systems with adequate response time to applications and final users.

In these networks contention is a classic problem. Contention occurs when two packets compete for the same resource (typically a link). As the channel capacity is limited, one of the packets will be transmitted, while the other will wait. If packets belonging to different flows request the same resource, contention will occur among all the packets, and flows will advance through the network at a lower pace. Moreover, contention may derive in severe congestion if the situation persists over time. In a congested situation, packets experience high latencies and the network collapses.

Traditionally, congestion has been managed by using two different strategies: proactive techniques and reactive techniques. Proactive techniques consists on preventing the formation of congestion. Data is injected into the network in such a way that congestion should never happen. This is achieved either by reserving in advance network resources (avoidance-based techniques) [18, 4] or by limiting the routes followed by packets (prevention-based techniques) $[1,16]$. In general, the use of both techniques implies a transmission scheduling that requires information about the occupancy of network resources (buffers, links, etc.) thus leading to inefficient solutions.

On the other hand, reactive techniques consist on detecting the congested situations and activating later some mechanisms to eliminate the congestion. Most of the proposed mechanisms $[17,8,3]$ consist on notifying congestion to the endnodes in order to cease or reduce the injection of packets.

However, recently, a totally different approach has been provided [12]. This new approach res on the idea that the congestion is not a problem by itself. Simply, there are too many packets competing for the same resource. The real problem is the negative effects introduced by the conges- 
tion. Once different flows become congested within the network (forming a congestion tree) they introduce HoL blocking to the rest of non-congested flows. HoL blocking occurs when a packet at the head of a queue can not be forwarded, and it blocks the rest of packets in the queue; even if those packets could make forward progress. If the HoL blocking were removed, then congestion would be harmless. Based on this approach, in [12] a new mechanism is provided, referred to as RECN. This mechanism detects congestion within the network, and instead of eliminating the congestion, it eliminates the HoL blocking introduced by the congested packets. This is achieved by dynamically allocating queues to the congested packets, thus separating them from the rest of packets. With this mechanism, maximum network throughput is achieved regardless of the presence of congestion. Unfortunately, RECN has been designed for Advanced Switching (AS) [15] as it relies on the use of source routing for checking if a packet belongs to a congested tree, which prevents RECN to be applied to other networks technologies such as InfiniBand (since InfiniBand uses distributed routing). Moreover, RECN requires some logic at the switches (queues and control structures) not available in commercial products.

\section{Motivation}

It is well known that VOQs at network level (referred in this paper to as $\left.V O Q_{n e t}\right)[2,14]$ is the best-known solution to the HoL blocking problem. In $V O Q_{n e t}$, every switch port implements as many queues as final destinations, and each queue is used only by packets addressed to a unique destination. Although $V O Q_{\text {net }}$ completely eliminates HoL blocking, it is not scalable at all, since memory requirements increase quadratically with the number of endnodes in the network. This situation is aggravated by the increasing demand for quality of service (QoS), as every service level requires a dedicated buffer per port.

An alternative is the use of VOQs at switch level (referred in this paper to as $V O Q_{s w}$ ). In $V O Q_{s w}$, each switch have at every input port as many queues as output ports, and packets are mapped according to the requested output port at the switch. Although the number of queues is significantly reduced, HoL blocking can still occur between flows sharing a subset of consecutive links along their paths. Indeed, in $[7,11]$ it was demonstrated that as network size increases, the percentage of HoL blocking not solved by $V O Q_{s w}$ increases.

In $[10,7,11]$ we proposed and evaluated an scalable and cost-effective solution to the HoL blocking problem, the Destination-Based Buffer Management (DBBM) mapping scheme. In DBBM packets are mapped to queues according to their destination (as in $V O Q_{n e t}$ ), however, the number of queues is significantly reduced (even lower than the ones used in $V O Q_{s w}$ ). Basically, DBBM makes that each queue store only packets for a subset of destination ports. This can be viewed as if the physical output ports of the network were grouped into a smaller set of logical output ports, and a queue was used at each switch to allocate only packets destined to a particular logical output port (i.e., VOQ at the logical port level). Therefore, most of the HoL blocking is eliminated (all the HoL blocking among different logical ports). The simplest implementation of DBBM is to use the lower bits of the destination ID of a packet to select the queue (we refer to this as modulo mapping). DBBM achieves a trade-off among implementation cost (the set of queues is reduced and the mapping function required at switches is straight forward) and efficiency (DBBM has demonstrated to be an efficient mapping scheme, obtaining much better performance than $V O Q_{s w}$ ).

Unfortunately, DBBM can still suffer from HoL blocking (among packets addressed to the same logic port). The percentage of affected destinations (victimized destinations) will depend on the number of queues. For example, with DBBM with 4 queues, only $25 \%$ of destinations will potentially be affected by the congested destination. Thus, as more queues were used in DBBM, the percentage of affected destinations would decrease.

Also, the percentage of HoL blocking not solved by DBBM depends on the number of congested destinations. If the number of congested destinations increases, potentially, more queues in DBBM will suffer HoL blocking, and the percentage of victimized destinations will increase. As an example, Figure 5.(e) shows the accepted traffic of each endnode in a $64 \times 64$ multistage network when random traffic is sent to all destinations and a hotpot is formed at endnode 30. DBBM with 8 queues was used. As can be observed, every eight destinations there is one with a significant lower reception rate. This is because of the HoL blocking introduced by the congested destination, thus introducing unfairness $\left(V O Q_{s w}\right.$ also suffers the same problem). Notice that this problem comes from the static nature of DBBM, because DBBM always maps the same destinations into the same queues.

In this paper we present an effective mechanism able to deal with HoL blocking problem as DBBM does but overcoming the DBBM fairness weakness. This mechanism will be also designed in such a way that can be used in InfiniBand without switch modifications.

The proposed mechanism, referred to as Dynamic DBBM (DDBBM), will eliminate most of the HoL blocking by using the DBBM technique, and whenever a destination becomes congested, its traffic will be dynamically separate; congestion flow will be separate from the non-congested one. In such a way, the unfairness introduced by DBBM will be totally eliminated.

Additionally, in this paper we will address the main aspects that must be dealt in InfiniBand in order to use DDBBM, without requiring any change to current switch architectures used in InfiniBand switches.

The rest of the paper is organized as follows. In Section 3 we describe the new proposed technique (DDBBM). Next, in Section 4 we describe how the mechanism can be applied 
to InfiniBand. Next, in Section 5 we analyze DDBBM in terms of scalability, throughput, robustness, and most important, fairness. Finally, we draw our conclusions in Section 6.

\section{Dynamic DBBM (DDBBM)}

DDBBM eliminates the HoL blocking not handled by DBBM (the one caused among packets addressed to the same set of destinations). Therefore, as DDBBM should be seen as an improvement of DBBM, all the good properties of DBBM still remain (most of the HoL blocking is eliminated with a low implementation complexity).

Basically, the DDBBM mechanism works as follows. When an endnode detects that itself is a congested destination, it notifies that situation to the sources sending traffic to it. Upon reception of the notification, sources will inject packets addressed to the congested destination with a bit activated (at the packet header). This bit is used by switches to separate congested flows from non-congested flows. In order to do this, at each switch input port one queue (referred to as the dynamic queue) is used to allocate the congested flows, whereas the remaining queues (referred to as DBBM queues) are used to allocate non-congested flows according to DBBM. Thus, each time a packet with its bit activated is received it is allocated at the dynamic queue. As an example, in a DDBBM mechanism with 8 queues for DBBM and a dynamic queue, a packet addressed to destination 48 will be mapped into queue zero (modulo mapping). However, if destination 48 becomes congested, then packets addressed to that destination will be mapped into the dynamic queue. As the congested packets will be mapped on a different queue, DBBM queues will not allocate congested packets and therefore, the HoL blocking that packets addressed to destination 48 might cause will be eliminated.

DDBBM resembles a limitation-based congestion control mechanism where destination nodes detect congestion and inform sources to limit the injection (to eliminate congestion). However, notice that DDBBM neither limits source traffic injection nor eliminates congestion. Instead, it will let the sources to keep their injection rate. A wellknown problem of limitation-based protocols is that their efficiency depends on network size and link bandwidth. Probably, at the moment sources are notified, the congestion may be formed within the network or may even vanished, thus introducing oscillations. However, this problem have a minor impact when DDBBM is implemented, as congestion will only affect to a small percentage of packets.

\subsection{Detection of Start/End of Congestion}

We need a fast and accurate detection mechanism at the endnode. The mechanism should be fast in order to minimize the time during which HoL blocking takes place. Also, the mechanism should be accurate in order to prevent false positive detections. Inaccurate detections could lead to worse results, since extra HoL blocking could be introduced. For instance, if the mechanism detects that two destinations are congested, one of them wrongly detected, massive HoL blocking would be introduced to the non-congested flow (as packets for both destinations would be mapped into the dynamic queue). Thus, the key piece of DDBBM is the congestion detection mechanism.

As a first approach to the detection mechanism, each endnode observes its received traffic rate. An endnode considers that it is becoming congested whenever its reception rate is higher than a fixed threshold value (Detection Threshold expressed in bytes, DT ). Similarly, an endnode is no longer congested whenever its reception rate is lower than a fixed threshold value (Low Threshold expressed in bytes, $L T$ ). The reception rate can be easily computed at the endnode. Simply the number of bytes received must be counted during a defined reception time frame (TimeFrame expressed in cycles).

With this mechanism, permanent congestion at a destination will be detected sooner or later depending on the $D T$ and $L T$ threshold values. However, experiencing a high reception rate does not necessarily means that the endnode is congested. Indeed, congestion occurs at a destination endnode only when there is more bandwidth demand than the offered bandwidth at the destination. Since the endnode only can count the quantity of bytes received, it does not know if the high reception rate is because of a congested situation (sources request more bandwidth than the offered one) or if it is because of sources request just the offered bandwidth. The only way to properly identify congestion would be achieved by inspecting switches and source endnodes. If they (switches and/or endnodes) start accumulating packets then there is a congested situation. However, notice that inspecting switches and endnodes would increase the complexity of the mechanism.

Instead, DDBBM will detect congestion at the endnodes by considering the number of sources (Number of sources threshold, $N S$ ) sending traffic and their injection rates (Source Injection Threshold, SIT). In case an endnode is congested, most of its received traffic will be generated by few sources. So, some sources will be injecting more traffic than the rest.

Figure 1 shows the detection mechanism with all the parameters to be considered. A register is used per each different threshold to be considered (DT, LT, NS , and $S I T)$. Also, additional registers are used for the time frame (TimeFrame) in cycles, the overall number of bytes received $(O R B)$, and a bank of registers for the number of bytes received per source $(S R B)$.

The mechanism works as follows. At the start of a time frame, the counter is set with the TimeFrame value and the $O R B$ and all the $S R B$ registers are set to zero. During the time frame, the counter is decremented cycle by cycle and the reception rate is quantified. Whenever a new packet arrives, the corresponding $S R B$ and the $O R B$ registers are updated accordingly. 
When the counter reaches zero a time frame has expired and the detection mechanism is triggered. At this point, the comparers are enabled. In particular, $O R B$ is compared with $D T$ register. Also, all the $S R B$ registers are compared in parallel with the $S I T$ register. Then, the number of $S R B$ registers with larger values than the $S I T$ register is calculated, and the result compared with the $N S$ register. With all this processing, the final decision on congestion is made. If the number of bytes received $(O R B)$ is higher than the $D T$ register and the number of sources sending too much data is higher than the $N S$ register, then the endnode is considered as congested. In that situation, the congestion bit $(C B)$ is set. However, if the number of bytes received $(O R B)$ is lower than the $L T$ register, the endnode is not considered congested anymore and the $C B$ bit is reset. Regardless of the detected situation, at this point in time a new time frame is started.

\subsection{Notification of Congestion}

Once an endnode detects the start or the end of congestion (the $C B$ bit changes), it notifies the new situation to that sources that have sent packets recently. This info is available in the detection mechanism logic ( $S R B$ register). This end-to-end notification mechanism keeps switch complexity low.

When end of congestion is detected, the endnode notifies only the sources that were previously notified about the congestion. Since the information located in the detection mechanism is reset each time frame, and the congested situation may last several time frames, the endnode needs a data structure to keep the information regarding the notified sources. For this reason, a notification bit vector is used at each endnode. The notification bit vector has as many elements (bits) as endnodes in the system. Once congestion is detected, the endnode sends a notification to all the endnodes that have sent traffic within the time frame ( $S R B$ register with a non zero value). At the same time, the corresponding bits in the notification vector are set. During the time the $C B$ bit is set, if a new source sends data to the endnode, it will be notified, and its notification bit will be set. When the end of congestion is detected $(C B$ bit is reset) a notification will be sent to those endnodes with their bits set in the notification vector. At the same time, the corresponding bits in the notification vector will be reset. DDBBM will use an specific control packet to notify the current congestion situation (referred to as a notification packet). Control packets uses the same resources than data traffic (notification in-band), thus consuming data bandwidth. In order to reduce to the minimum the consumed bandwidth, notification packets are only packet header (without payload).

\subsubsection{DDBBM on a Sender Endnode}

Each endnode will have a bit vector (referred to as status bit vector) with as many bits as endnodes on the network. In the status bit vector, each bit will correspond to an endnode status. When the bit for an endnode is set, it means that the endnode is considered as a congested destination. Initially all the endnodes are considered as non-congested endnodes. When an endnode receives a notification packet (meaning start or end of congestion) it will commute the bit of the endnode that sent the notification. Thus, the congestion bit mirrors the $C B$ bit at destination.

When an endnode has data to transmit, a data packet is built and injected. The value of the congestion bit on the packet header is stamped directly from the status bit vector. Therefore, all the packets addressed to a congested destination will have activated their congestion bit.

\subsubsection{DDBBM on a Switch}

Switches must identify packets going to a congested destination in order to separate them from the rest of traffic. Congested flows must be allocated in the dynamic queue, while the other flows must be allocated in the DBBM queues. The allocation decision is taken based on the congested bit of the header of each arriving packet. If the bit is reset, the packet is allocated in a DBBM queue (DBBM modulo mapping). However, if the bit is set, the received packet is allocated in the dynamic queue.

The dynamic queue can be flow controlled in the same way as the other queues are. Indeed, this queue can be implemented in the same memory. Thus, credit-based flow control can be used

\subsubsection{Out of order delivery issues}

Since traffic will be dynamically separated, out of order issues may appear. Out of order may be present only among packets for the same flow (source-destination pair). Since such packets are mapped into the same queues (DBBM strategy) they will arrive at the destination in the same order as they were injected. However, now in DDBBM, two consecutive packets injected from the same source to the same destination may arrive out of order, simply because the second packet is separated from the normal traffic and thus mapped in the dynamic queue, thus potentially advancing at a faster rate than the previous packet.

In order to guarantee in order delivery, the sender must ensure that packets belonging to the same message will be handled in the same way. For this reason, if the sender receives a notification while a message is being sent (some packets belonging to that message have been already sent), it will not modify the congestion bit in the remaining packets of that message. The new value will be applied to the next message. Notice that only a fixed delay is introduced in the sender. 

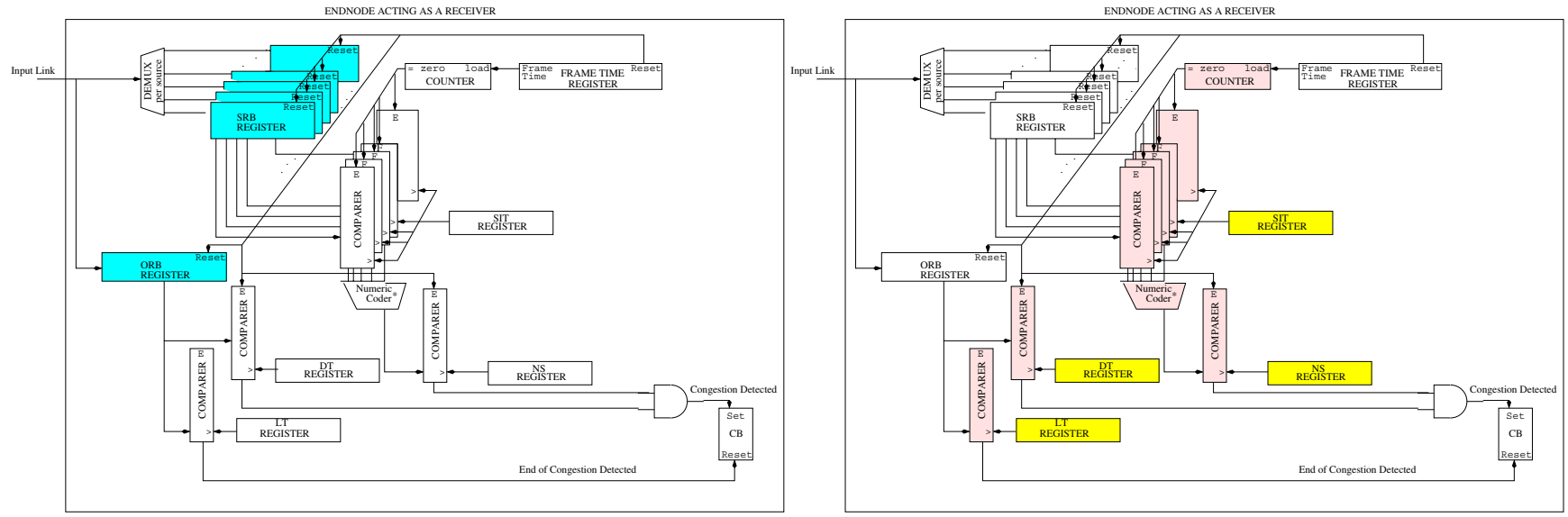

Figure 1. Logic for measuring congestion and detecting congestion.

\section{Implementation of DDBBM on InfiniBand}

One of the goals of the paper is to adapt DDBBM to InfiniBand (IBA) with no switch modification. Indeed only tables provided by the standard and located at switches will be modified.

In IBA, routing and virtual channel (they are referred to as Virtual Lanes, VLs) selection is performed based on the destination local ID (DLID) and the service level (SL) fields of the packet header. These two fields are computed at the source node and do not change along the path. Every switch has a forwarding table which provides only one output port (and always the same) for each destination. Thus, IBA uses deterministic routing.

Up to 15 data virtual lanes can be implemented in IBA. Virtual lane selection is based on the use of service levels (SLs). By means of SLtoVL mapping tables located on every switch, SLs are used to select the proper VL at each switch. This table returns, for a given input port and a given SL, the VL to be used at the corresponding output port. For this, the SL is placed at the packet header and it cannot be changed by the switches. Therefore, we should also assign the proper SL that must be used for a given path. VLs and SLs were initially defined in IBA for providing QoS, deadlock avoidance and traffic parting.

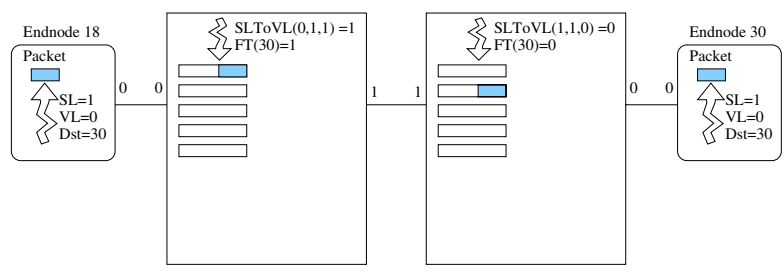

Figure 2. Example of the VLs used in IBA.
Figure 2 shows a routing example in InfiniBand. Whenever a packet is injected into the network, the endnode (endnode 18) computes the SL of the packet (there are up to $16 \mathrm{SLs}$ ) and the initial VL to use. In the example, the SL is set to 1 and the initial VL is set to 0 . At the first switch the VL used to map the packet is VLO (as the initial VL was set to zero). At that switch the next VL to use might be changed if the SLtoVL indicates so. In particular, the SLtoVL table at the first switch indicates that at the next switch the VL to use will be VL1. Notice also that the output port selected at each switch is extracted from the forwarding table (FT function in the example).

To implement DDBBM we will use SL identifiers and different VLs. A particular VL (referred to as $V L_{i}$ ) can be used along any path by using a SL identifier (referred to as $\left.S L_{i}\right)$ and all the SLtoVL tables being programmed in such a way that whenever the SL of the packet is $S L_{i}$, regardless of the input and output ports, the provided VL is $V L_{i}$. Thus, if we want to implement DDBBM with 5 queues (4 queues for DBBM, and one for the dynamic queue) we must reserve 5 SL identifiers and program the corresponding SLtoVL tables.

Now, at the endnodes we need to compute for each packet the proper SL. We need to implement the status bit vector and the modulo selection at the endnode. For efficiency reasons the vector should be included at the network interface. Simply whenever a packet is going to be injected, the status bit for the corresponding destination is inspected. If the bit is set, then the SL that forces the dynamic queue ( $S L_{4}$ for a DDBBM implementation with 5 queues (VL0 through VL4)) is selected. If not, then lower bits of destination indicate the SL. In our example, the lower two bits. Notice that the selection may be implemented in the subnet manager located on every channel adapted in InfiniBand.

Finally, we need to implement the detection mechanism at the endnodes and the notification of congestion. Regard- 
ing the detection mechanism, it must be implemented at the network interfaces. Again, at the endnodes we may rely on the subnet manager in order to keep the statistics of traffic and to issue notification packets to the endnodes. For notifications we can use the VL15. This virtual lane is reserved for control packets in InfiniBand.

\section{Performance Evaluation}

In this section we will evaluate the DDBBM strategy. For this purpose we have developed a detailed event-driven simulator that allows us to model the network at the register transfer level. Firstly, we will describe the main simulation parameters and the modeling considerations we have used in all the evaluations. Secondly, we will present the evaluation results and analyze them.

\subsection{Simulation Model}

The simulator models an ICTN with switches, nodes, and links. Buffers up to $1 \mathrm{~KB}$ are modeled for both the input and the output ports of every switch. The buffer capacity is statically divided by the number of queues defined by each of the evaluated mapping schemes, resulting in a fixed size per queue.

At every switch packets are forwarded from any input queue to any output queue through a multiplexed crossbar. We have considered a crossbar bandwidth of $1.5 \mathrm{~GB} / \mathrm{s}$ with a speedup of 1.5. The crossbar is controlled by a scheduler that receives requests from the packets at the head of any input queue. A requesting packet is forwarded only if the corresponding crossbar input and crossbar output are free. At each output port a weighted round-robin arbiter selects the output queue to be served.

For links we assume serial full-duplex pipelined transmissions with $1 \mathrm{~GB} / \mathrm{s}$ effective bandwidth. The link-level flow control (LL-FC) protocol is credit-based; a packet can be transmitted downstream only if a credit is available. Whenever a packet frees an input buffer location a new credit is sent to the output port upstream. A similar flow control scheme has been implemented for the internal switch traversal (input-output packet forwarding). The maximum number of credits per output (input) port depends on the buffer size at the next input (output) port and the total number of queues. The LL-FC packets share the link bandwidth with data traffic.

The endpoints are connected to switches using Input Adapters (IAs). Every IA is modeled by (i) a fixed number $N$ of message admittance queues organized in VOQ; (ii) and a variable number of injection queues organized similarly to the output ports of a switch. When a new message is generated, first it is stored completely in the admittance queue assigned to its destination; then it is segmented into 64B packets before being transferred to an injection queue. The transfer from admittance queues to injection queues are controlled by a round-robin arbiter. The transmission of packets from injection queues into the network is controlled by a weighted round-robin arbiter.

\subsection{Topologies and Traffic Patterns}

DDBBM will be evaluated in different bidirectional multistage networks (BMINs) and 2D/3D meshes. In particular, $64 \times 64,512 \times 512$, and $1024 \times 1024$ BMINs will be used, each one built using 8-port switches interconnected in a perfect shuffle connection pattern. The routing algorithm is deterministic. For the regular topologies, $4 \times 4,8 \times 8$, $16 \times 16,8 \times 8 \times 4$, and $4 \times 4 \times 4$ will be evaluated. In this case we will use the Dimension Order Routing (DOR).

For the BMINs networks we have defined 7 different synthetic traffic patterns (see Table 1 ). In $\# 1 \mathrm{~B}^{1}$ all the sources inject at $100 \%$ rate with an uniform destination distribution. Cases \#2B-\#7B cause at least a congestion tree by oversubscribing a hotspotted endnode: $30 \%$ of sources injecting at the full rate to a randomly selected hotspot destination. The rest of traffic (background traffic) is made of the remaining sources (70\%) injecting at $60 \%$ of link bandwidth to randomly selected destinations. As the background traffic shares links and queues with the flows belonging to the congestion tree, substantial HoL blocking will be introduced in multiple switches.

On the other hand, in regular networks we have defined 9 different scenarios based on synthetic traffic patterns (see Table 1). All the cases cause a congestion tree by oversubscribing the hotspotted endnode. In this case, the background traffic is made of $70 \%$ of the sources injecting at $20 \%$ of link bandwidth to randomly selected destinations, while the remaining $30 \%$ of sources inject at full rate to a randomly selected hotspot destination.

We will analyze the behavior of DDBBM with different number of queues. For comparison purposes we will also evaluate the $V O Q_{n e t}, V O Q_{s w}$, and the DBBM mapping policies. For DBBM we will also use different number of queues.

\subsection{DDBBM Sensitivity to Thresholds}

In order to evaluate the robustness of DDBBM to changes in DT, LT, and SIT parameters, cases \#1B, \#3B, $\# 2 \mathrm{M}$ and \#5M will be evaluated with the following values of these parameters: $D T=0.8,0.95, L T=0.2,0.4$, and $S I T=0,6$. The $D T$ and $L T$ parameters are expressed as percentages of the reception rate. However, the SIT parameter is expressed as the times that the source exceeds the base injection rate $(100 / \mathrm{N}) \%$ (assuming a network with $N$ endnodes). For instance, a $S I T=6$ means that the destination must

\footnotetext{
${ }^{1}$ Notice that in order to differentiate clearly the studied cases, BMINs cases will be referred to as the case number followed by a B letter while mesh cases will be referred to as the number of the case followed by an M letter.
} 


\begin{tabular}{|c|c||c||c|}
\hline \multicolumn{2}{|c|}{ Traffic to: } & random dests & hotspot \\
\hline Case & $\begin{array}{c}\text { Network } \\
\text { Evaluated } \\
\text { (endnodes) }\end{array}$ & $\begin{array}{c}\text { \% of injecting } \\
\text { sources (IR) }\end{array}$ & $\begin{array}{c}\% \text { of injecting } \\
\text { sources (IR) } \\
\text { [destinations] }\end{array}$ \\
\hline \#1B & $64 \times 64(4)$ & $100 \%(100 \%)$ & $-(-)[-]$ \\
\hline \#2B & $64 \times 64(4)$ & $70 \%(60 \%)$ & $30 \%(60 \%)[1]$ \\
\hline \#3B & $64 \times 64(4)$ & $70 \%(60 \%)$ & $30 \%(100 \%)[1]$ \\
\hline \#4B & $64 \times 64(4)$ & $70 \%(90 \%)$ & $30 \%(60 \%)[1]$ \\
\hline \#5B & $64 \times 64(4)$ & $70 \%(60 \%)$ & $30 \%(100 \%)[5]$ \\
\hline \#6B & $512 \times 512(4)$ & $70 \%(60 \%)$ & $30 \%(60 \%)[1]$ \\
\hline \#7B & $1024 \times 1024(4)$ & $70 \%(60 \%)$ & $30 \%(60 \%)[1]$ \\
\hline \hline \#1M & $8 \times 8(1)$ & $100 \%(20 \%)$ & $-(-)[1]$ \\
\hline \#2M & $4 \times 4(4)$ & $100 \%(20 \%)$ & $-(-)[1]$ \\
\hline \#3M & $8 \times 8(1)$ & $70 \%(20 \%)$ & $30 \%(100 \%)[1]$ \\
\hline \#4M & $8 \times 8 \times 4(1)$ & $70 \%(20 \%)$ & $30 \%(100 \%)[1]$ \\
\hline \#5M & $4 \times 4(4)$ & $70 \%(20 \%)$ & $30 \%(100 \%)[1]$ \\
\hline \#6M & $16 \times 16(1)$ & $70 \%(20 \%)$ & $30 \%(100 \%)[1]$ \\
\hline \#7M & $8 \times 8(4)$ & $70 \%(20 \%)$ & $30 \%(100 \%)[1]$ \\
\hline \#8M & $4 \times 4(16)$ & $70 \%(5 \%)$ & $30 \%(100 \%)[1]$ \\
\hline \#9M & $4 \times 4 \times 4(4)$ & $70 \%(20 \%)$ & $30 \%(100 \%)[1]$ \\
\hline
\end{tabular}

Table 1. Topologies and traffic patterns.

receive $(600 / N) \%$ of the total received traffic from an particular source to decide that this source is contributing to the congestion. A value of zero means that the SIT parameter is disabled.

For all the cases, the $N S$ parameter has been set to one. Thus, at minimum the traffic injected by at least two sources must overtake the SIT threshold to congestion be detected.

\subsubsection{Evaluation Results}

Figure 4.(a) shows results for the \#1B topology/traffic case with $D T=0.95, L T=0.2$, and $S I T=0$. In this case, all the evaluated mapping schemes except DDBBM achieve the maximum throughput. In this case, as the $S I T$ parameter is disabled, most of the endnodes detect congestion (although uniform traffic is in the networks). All the endnodes receiving at a rate higher $95 \%$ of their link bandwidth will be considered as a hotspotted endnode. These detections can be considered as false positives. After notifying sources, all the injected packets are mapped in the dynamic queue thus introducing massive HoL blocking. Therefore, DDBBM exhibits poor performance levels. We have analyzed topology/traffic case \#1B with increasing values of $S I T$. Results show that as we increase the value of $S I T$, DDBBM performance increases. Concretely, when $S I T$ is set to 6 , DDBBM achieves the same performance as $V O Q_{n e t}$, as the false congestion detected by endnodes is filtered. Figure 4.(b) shows results for topology/traffic case \#1B with $S I T=6$.

Moreover, we have analyze the impact of $D T$ and $L T$ thresholds. Results obtained are similar for all the $D T$ and $L T$ defined values, showing that $D T$ and $L T$ thresholds have low impact on network performance.

On the other hand, the same evaluation applied to topol-

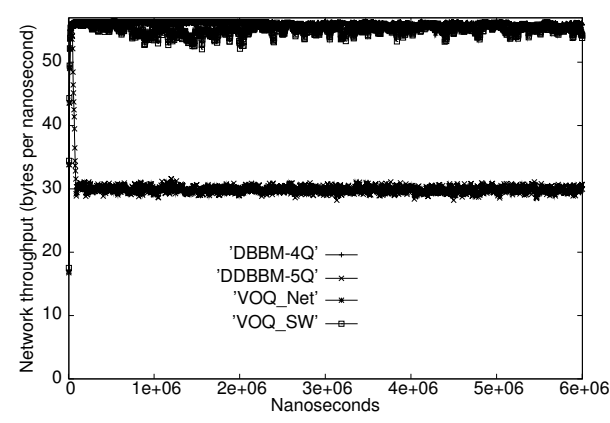

Figure 3. Accepted traffic. $S I T=0$

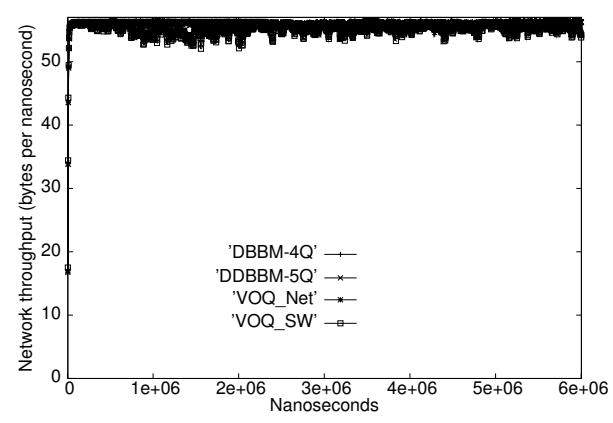

Figure 4. Accepted traffic. $S I T=6$

ogy/traffic cases \#3B, \#2M and \#5M, not shown, does not present any difference in the obtained performance for case \#1B.

Therefore, we can conclude that the DDBBM mechanism is largely insensitive to $D T, L T$, and under high uniform traffic and BMIN topologies there exists a dependence on the SIT parameter. Based on these results, in the following evaluations we will use the following DDBBM parameters: $D T=0.95, L T=0.2$, and $S I T=6$.

\subsection{Fairness of DDBBM}

In this Section we will analyze the level of unfairness introduced by the different mapping policies. From previous works we know that DBBM introduces certain levels of unfairness to some destinations. This is because DBBM maps different flows to the same queue, and thus HoL blocking is not eliminated completely. Notice that this problem is the main motivation for designing DDBBM.

In Figure 5 we can see the traffic received by each endnode for topology/traffic case \#3B $(64 \times 64 \mathrm{BMIN})$ when $V O Q_{n e t}, V O Q_{s w}$, DBBM-4Q and DDBBM-5Q schemes are used. The endnode with the highest traffic reception rate corresponds to the hotspot (endnode 30), which reaches $90 \%$ of reception rate (axes are truncated at $50 \%$ ). With $V O Q_{n e t}$ every destination, except the hotspot, receives roughly the same goodput. However, with $V O Q_{s w}$, all the flows that share any queue along their path with the 

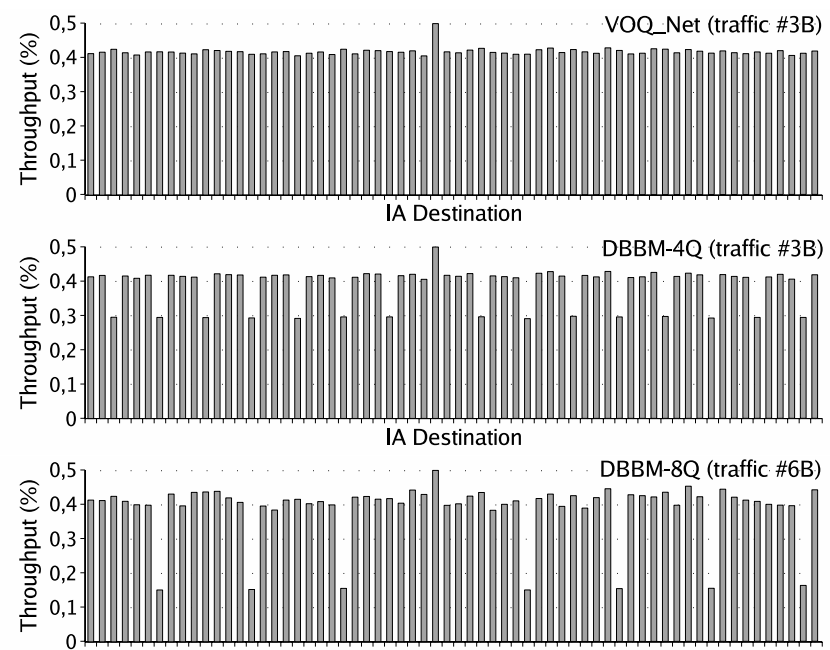

IA Destination

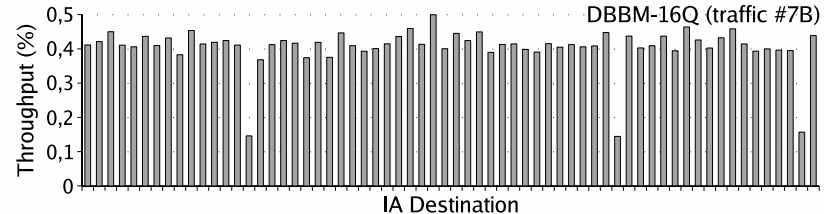

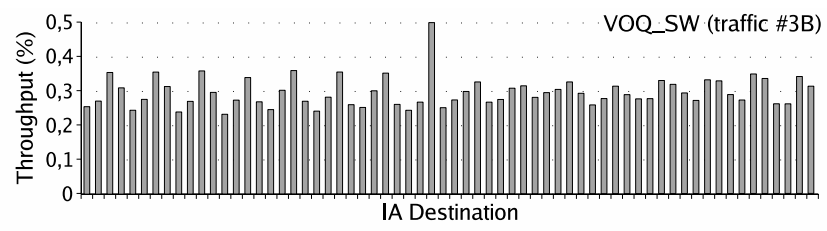
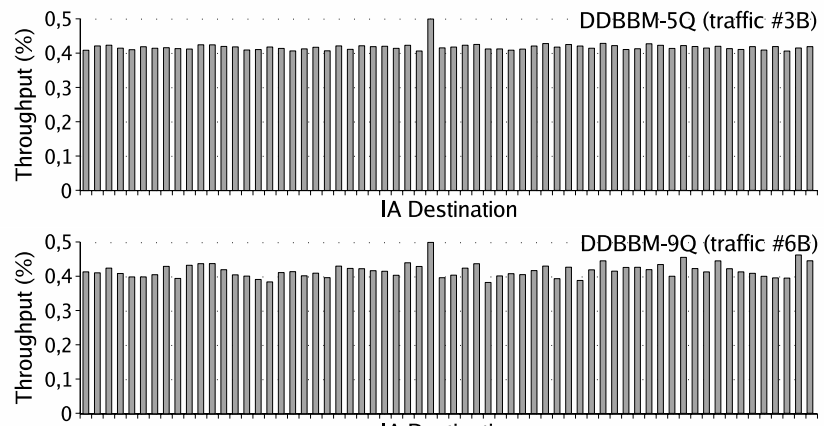

IA Destination

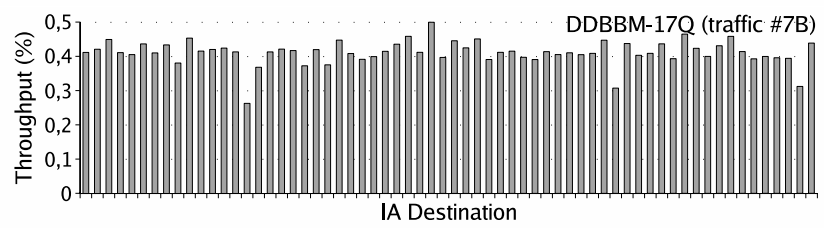

Figure 5. BMIN Accepted traffic per destination.

congested flow suffer from HoL blocking. The bar graph of the $V O Q_{s w}$ in Figure 5, we can see how every 4 consecutive endnodes exhibit similar percentages of accepted traffic. This pattern is consequence of the number of links per switch together with the routing algorithm applied. The routing algorithm will determine the output link for each packet and consequently which packets will be mapped into the same queue as the packets addressed to the congested destination. Hence the reduction in the number of received packets by the victimized destinations. With DBBM (DBBM-4Q bar graph in Figure 5) the number of affected flows depends on the number of queues, but not on routing neither the number of links per switch.

However, DDBBM-5Q completely eliminates the HoL blocking, and hence the unfairness. The DDBBM-5Q bar graph in Figure 5 shows that the traffic received by each endnode is roughly the same. Therefore, the HoL blocking experienced by DBBM has been completely eliminated by DDBBM. Thus, the detection mechanism has identified correctly the congested destination (endnode 30) and the packets addressed to the congested destination have been mapped into the dynamic queue.

Bar graphs of DBBM-8Q/16Q and DDBBM-9Q/17Q in Figures 5 show similar results with topology/traffic cases \#6B and \#7B. In these cases, the mechanism is much more stressed as the network is larger $(512 \times 512$ and $1024 \times 1024$ BMIN networks). However, only in case \#7B $(1024 \times 1024$ BMIN) DDBBM shows some kind of unfairness. This is because although congestion tree is isolated, the reduced number of queues applying DDBBM scheme respect to the total number of endnodes will introduce a slight HoL blocking among the non congested flows.

To conclude, results show that only when packets addressed to the congested destination are isolated in a queue, unfairness is eliminated. Excepting $V O Q_{n e t}$ and DDBBM schemes, all the other schemes introduce some degree of unfairness under high load and congestion. However, DDBBM with a very low number of queues eliminates unfairness completely.

Fairness has been also analyzed in 2D/3D meshes in order to confirm the good behavior of DDBBM observed in BMIN networks. In this case, similar results have been obtained. Figure 6 shows received traffic for each destination over the different mapping schemes $\left(V O Q_{n e t}, V O Q_{s w}\right.$, DBBM, and DDBBM, respectively) for topology/traffic cases \#5M ( $4 \times 4$ mesh with 64 endnodes), and \#8M $(4 \times 4$ mesh with 256 endnodes). In all the analyzed cases (cases \#5M, \#7M (figures not shown), \#8M, and \#9M (figures not shown)) DDBBM successfully eliminates dynamically all the unfairness that would be introduced by the congested destination. This behavior confirms that DDBBM independently of the size and topology of the network does not present unfairness.

\subsection{Throughput Analysis}

Finally we evaluate DDBBM in terms of achieved network throughput. Figure 7 shows the obtained through- 

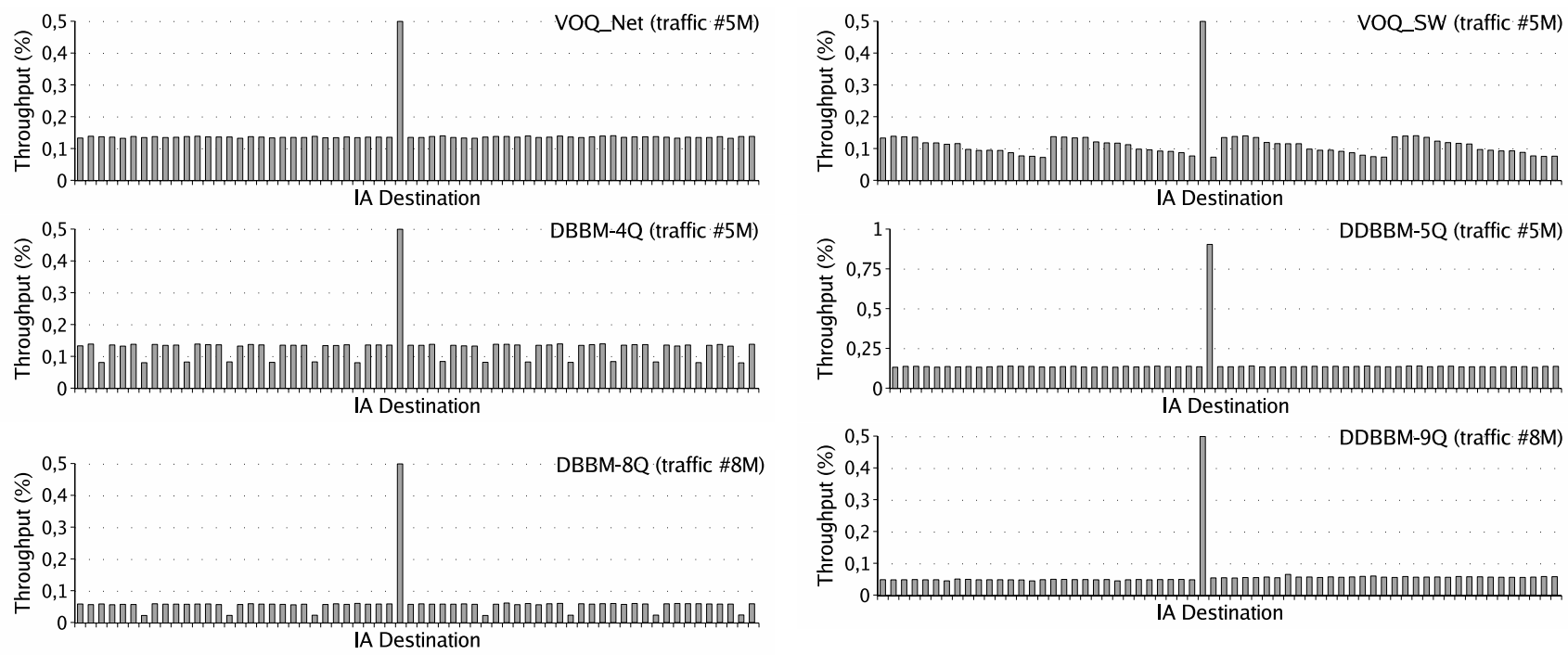

Figure 6. Mesh Accepted traffic per destination.

put in the evaluated cases. The graph on top in Figure 7 shows the throughput achieved for topology/traffic cases \#2B, \#3B, \#4B, and \#5B. For topology/traffic cases \#2B we can observe that both $V O Q_{\text {net }}$ and DDBBM-5Q achieve the maximum throughput whereas DBBM-4Q and $V O Q_{s w}$ show lower throughput numbers. $V O Q_{s w}$ achieves $70 \%$ of the DDBBM throughput, whereas DBBM-4Q performs better. Concretely, DBBM-4Q achieves $92 \%$ of the DDBBM throughput. Similarly, for topology/traffic cases \#3B and \#4B, where DDBBM-4Q performs equal than $V O Q_{n e t}$, and DBBM-4Q achieves $88 \%$ and $89 \%$ of DBBM throughput (cases \#3B and \#4B respectively), whereas $V O Q_{s w}$ only achieves 59 and $61 \%$ of DDBBM throughput (cases \#3B and \#4B respectively). Finally, in case \#5B, where 5 lower intense congestion trees are formed, all of the evaluated mapping schemes perform the maximum throughput. As a summary of all these results: DDBBM-5Q achieves $V O Q_{n e t}$ throughput in all the hot spot evaluated cases.

For 2D meshes, the bar graph down in Figure 7 shows the throughput achieved for topology/traffic cases \#1M, \#3M, \#4M, and \#6M. We see that topology/traffic cases \#1M and \#2M show equal characteristics. All the mapping schemes achieves the maximum performance. This is because the network is able to absorb the injected traffic at the rate that is injected (not hotspotted endnode appears). However, when a congestion tree is formed, as in cases \#3M, $\# 4 \mathrm{M}$, and \#6M, results show that only DDBBM is able to achieve $V O Q_{\text {net }}$ throughput. Likewise, we see that although $\mathrm{DBBM}$ is not able to reach $V O Q_{\text {net }}$ throughput, it shows better performance than $V O Q_{s w}$ as this method perform the worst in all the cases. Notice that this behavior keeps despite the increase in the number of endnodes (cases \#4M and \#6M). These results confirms our results from BMIN networks: regardless of the topology DDBBM is able to reach maximum performance while using a reduced set of queues.

It has to be noted that we also have evaluated the DDBBM mechanism with real traffic (extracted from IP and $\mathrm{SAN}$ traces). In all the cases, the DDBBM mechanism has been achieved the maximum performance with very low queue requirements. It also completely eliminates the unfairness introduced by DBBM. However, we believe that results from the synthetic traffic are much more interesting since we can predict the results.

\section{Conclusions}

In this paper we have proposed a mechanism able to dynamically eliminate the HoL blocking caused by congested endnodes. This is achieved by designing a detection mechanism at the endnodes and then notifying sources in order to separate the traffic sent to congested endnodes from the rest of traffic. The mechanism has been designed in order to simplify the switch design. Therefore, it allows its use on commercial products. Indeed, the paper has presented also a method to apply DDBBM in InfiniBand without hardware modification.

The results presented clearly demonstrate that the proposed scheme, DDBBM, achieves similar performance than $V O Q_{n e t}$ scheme, and better than the other analyzed schemes (DBBM and $V O Q_{s w}$ ), with much fewer resources. These good results are in terms of throughput and fairness, under a wide range of traffic and topologies. Also, DDBBM sensitivity to threshold values has been evaluated. Results have shown that only under high uniform traffic and BMIN topologies there exists a dependence on the SIT parameter. However, with a fine tuning of the parameter, the 

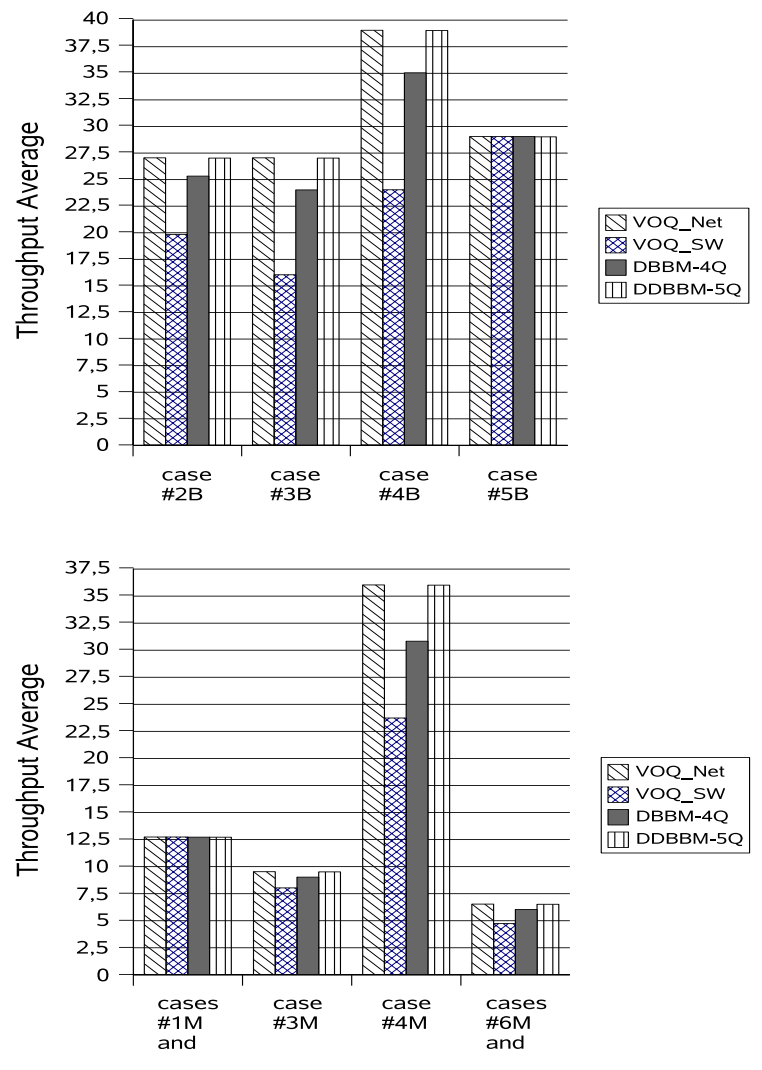

Figure 7. Throughput vs Mapping Scheme.

adverse case is solved. Also, detection thresholds ( $D T$ and $L T$ ) are highly insensitive to DDBBM final performance.

As future work we plan to evaluate the DDBBM mechanism on real traffic. For this we plan to implement the mechanism on InfiniBand and to use real InfiniBand platforms with real traffic for its test.

\section{References}

[1] G. S. Almasi and A. Gottlieb, "Highly parallel computing," Ed. Benjamin-Cummings Publishing Co., Inc., 1994.

[2] T. Anderson, S. Owicki, J. Saxe, and C. Thacker, "High Speed Switch scheduling for local area networks," in ACM Trans. Computer Systems, Nov. 1993.

[3] E. Baydal and P. Lopez, "A Robust Mechanism for Congestion Control: INC," in Proc. 9th International Euro-Par Conference, pp. 958-968, Aug. 2003.

[4] R. Bianchini, T. J. LeBlanc, L. I. Kontothanassis, and M. E. Crovella, "Alleviating Memory Contention in Matrix Computations on Large-Scale Shared-Memory Multiprocessors," Tech. report 449, Computer Science Dept., Rochester University, April 1993.
[5] IBM BG/L Team, "An Overview of BlueGene/L Supercomputer," in High Performance Networking and Computing (SC2002), Nov. 2002.

[6] N.J. Boden et al, "Myrinet - A gigabit per second local area network," in IEEE Micro, Feb. 1995.

[7] T. Nachiondo, J. Flich, and J. Duato, "Efficient Reduction of HOL blocking in Multistage Networks," in Proc. 2005 Int. Parallel and Distributed Processing Symposium, April 2005.

[8] W. J. Dally and H. Aoki, "Deadlock-Free Adaptive Routing in Multicomputer Networks Using Virtual Channels," in IEEE Trans. on Par. and Distr. Systems, vol. 4, no. 4, April 1993.

[9] Earth Simulator Center. http://www.es.jamstec.go.jp/ esc/eng/index.html.

[10] J. Duato, J. Flich, and T. Nachiondo, "Cost-Effective Technique to Reduce HOL-blocking in Single-Stage and Multistage Switch Fabrics," in Proc. Euromicro Conf. on Par., Distr. and Network-based Processing, Feb. 2004.

[11] T. Nachiondo, J. Flich, J. Duato, and M. Gusat, "Cost/Performance Trade-offs and Fairness Evaluation of Queue Mapping Policies," in Proc. International Euro-Par Conference, Lisbon, Aug. 2005.

[12] J. Duato, I. Johnson, J. Flich, F. Naven, P. Garca, and T. Nachiondo,"A New Scalable and Cost-Effective Congestion Management Strategy for Lossless Multistage Interconnection Networks," in Int. Symp. on High-Performance Comp. Arch., Feb. 2005.

[13] InfiniBand Trade Association, "InfiniBand Architecture. Specification Volume 1. Release 1.0," Available at http://www.infinibandta.com/.

[14] N. McKeown, "Scheduling algorithms for inputqueued cell switches," Ph.D. Thesis, University of California at Berkeley, 1995.

[15] "Advanced Switching for the PCI Express Architecture," White paper.

[16] S. L. Scott, and G. S. Sohi, "The Use of Feedback in Multiprocessors and Its Application to Tree Saturation Control," in IEEE Trans. on Parallel Distr. Systems, vol. 1, no. 4, Oct. 1990.

[17] M. Thottethodi, A. R. Lebeck, and S. S. Mukherjee, "Self-Tuned Congestion Control for Multiprocessor Networks," in Proc. Int. Symp. High-Performance Computer Architecture, Feb. 2001.

[18] P. Yew, N. Tzeng, and D. H. Lawrie, ”Distributing Hot-Spot Addressing in Large-Scale Multiprocessors," in IEEE Trans. Computers, vol. 36, no. 4, April 1987. 